\title{
Carbapenemase-producing Klebsiella pneumoniae in elderly frail patients admitted to medical wards
}

\author{
Antonio Nouvenne, Andrea Ticinesi, Tiziana Meschi \\ Department of Clinical and Experimental Medicine, University of Parma, Italy
}

\begin{abstract}
Carbapenemase-producing Klebsiella pneumoniae (CPKP) is rapidly emerging as a leading nosocomial infection in many countries, especially in Italy where it is considered endemic. In this paper we briefly summarize the main characteristics of this outbreak, focusing on its recent spread to elderly frail patients admitted to internal medicine and geriatric wards. Severity of disease, clinical complexity and a large number of comorbidities seem to be major risk factors in clinical practice, although scientific evidence is still lacking, since until now studies have been carried out mainly in intensive care settings. We also discuss the possible role of gut microbiota in CPKP colonization onset and the possible role of pre-probiotics in promoting eradication.
\end{abstract}

\section{Introduction}

Nosocomial infections by multi-drug resistant Gram-negative bacteria have nowadays become a worldwide threat for the health of hospital inpatients. ${ }^{1}$ In this scenario, carbapenemase-producing Klebsiella pneumoniae should be considered a major health concern, since it has the capacity of causing large pan-

Correspondence: Antonio Nouvenne, Department of Clinical and Experimental Medicine, University of Parma, via A. Gramsci 14, 43126 Parma, Italy.

Tel.: +39.0521.703626 - Fax: +39.0521.940993

Mob.: +39.349.2258317.

E-mail: antonio.nouvenne@alice.it

Key words: Carbapenemases; Klebsiella pneumoniae; elderly patients; comorbidities; gut microbiota.

Contributions: AN, global design, text editing, pilot study design and fulfilment; AT, literature review and text editing; TM, global design and critical review.

Conflict of interest: the authors declare no potential conflict of interest.

Part of the topics discussed in this paper were presented at the $12^{\text {th }}$ European Congress of Internal Medicine (European Federation of Internal Medicine EFIM), Prague, 2-5 October 2013 , in the infectious disease oral presentation session.

Received for publication: 20 January 2014.

Accepted for publication: 27 January 2014.

This work is licensed under a Creative Commons Attribution NonCommercial 3.0 License (CC BY-NC 3.0).

CCopyright A. Nouvenne et al., 2015

Licensee PAGEPress, Italy

Italian Journal of Medicine 2015; 9:116-119

doi:10.4081/itjm.2014.476 demic foci in different hospital settings. ${ }^{2}$ Carbapenemases are special types of $\beta$-lactamase that show high genetic variability and relevant hydrolyzing capacity for carbapenems. K. pneumoniae is the most frequent species where the production of carbapenemases has been documented, even if reports of other bacterial species showing this mechanism of resistance to carbapenems, like Acinetobacter baumannii and Citrobacter freundii, are constantly rising. ${ }^{2-5}$

\section{Epidemiology}

The first in vivo isolation of a carbapenemase-producing $K$. pneumoniae (CPKP) strain dates back to 2000 in North Carolina in an intensive care unit patient. ${ }^{6}$ In the following years, many CPKP-related cases of severe septic shock have been described in Intensive Care Units (ICUs) of Northeastern United States. ${ }^{7}$ By the end of 2000s, CPKP had reached all developed countries worldwide, but characteristically affecting different countries in a non-homogeneous way. In fact, there are some countries in Europe, like Greece, where CPKP infection is endemic by now, while, for example, in Portugal and Sweden it has been detected only sporadically. ${ }^{2,3}$

Italy has faced CPKP later than other European countries, but, if possible, in a more serious way. The first isolation is dated 2009 in Florence in a patient with a complicated intra-abdominal infection ${ }^{8}$ and, since then, CPKP has quickly spread all over the country with a large number of pandemic outbreaks, so that Italy has been recently upgraded to an endemic country status. ${ }^{2}$

\section{Clinical features}

CPKP primarily colonizes lower intestinal tract and inguinal or perineal skin of hospital inpatients. Colo- 
nization is generally asymptomatic and does not require specific treatment. However, in some cases, CPKP can enter bloodstream and cause systemic infections, mainly complicated urinary tract infections or septic shock, whose prognosis is generally unfavorable even when an adequate intensive medical treatment is instituted. Mortality rates of about $41-80 \%$ have been reported in literature when infection occurs. ${ }^{910}$ Given the high degree of antibiotic resistance by these bacteria, therapeutic options are indeed very limited, including variable combinations of aminoglycosides, tigecycline, colistin and fosfomycin. There is much debate in literature about the possible use of carbapenems at high dosage or in combination therapy when $K$. pneumoniae minimum inhibitory concentrations for imipenem are $<4 \mathrm{mg} / \mathrm{L}$. Pros of this approach are the drug-sparing policy and the low risk for further resistance induction, cons are the controversial efficacy and the low tolerability of high-dose regimens. ${ }^{11}$ However, there is also growing evidence that combination therapy, for example with meropenem, colistin and tigecycline, is the only effective way to diminish mortality. 12,13

\section{Transmission}

CPKP transmission is mainly by contact, either direct or indirect. Subjects with a normal intestinal microbiota, like healthcare professionals but unlike hospital inpatients, are generally protected against colonization, even if they may act as vehicles and thus transmit bacteria to susceptible patients. Sanitary measures such as contact isolation, cohorting of positive patients and epidemiologic surveillance with weekly rectal swabs performed to all high-risk patients of the same ward of the index case have proven to be effective in limiting CPKP transmission..$^{14,15}$
Few studies have evaluated risk factors for colonization or infection so far. In ICU-admitted patients, the importance of prior antibiotic exposure, prior hospitalization, severe illness and respiratory failure has been emphasized, ${ }^{16,17}$ together with bacterial genotype and phenotype. ${ }^{18}$

\section{New clinical scenarios and perspectives}

In the existing literature, $\mathrm{CPKP}$ infection is mainly described as an ICU-setting concern. However, clinical practice, at least in Italy, is rapidly changing. CPKP outbreaks are seen more and more often also in internal medicine and geriatric wards, especially in frail elderly subjects with multiple comorbidities. There are actually some epidemiological reports showing that most CPKP patients are not infected in an ICU-setting, but instead in common internal medicine wards, and data seem to show that this phenomenon is continuously rising as the diffusion of CPKP spreads. ${ }^{19-21}$ As a matter of fact, besides published data, CPKP is rapidly emerging as one of the leading nosocomial infections in internal medicine practice and rectal colonization is more and more often recognized anywhere an epidemiologic surveillance program is instituted.

Susceptibility to nosocomial infections and immunologic rearrangements globally known as immunosenescence are well-known features of frailty. ${ }^{22}$ On the other side, CPKP isolates in everyday clinical practice do more and more often belong to elderly with a high degree of clinical complexity, a large number of comorbidities and frequently bedridden or cognitively impaired. Thus we may hypothesize that frailty and comorbidity themselves are a strong risk factor for CPKP colonization, together with the ones already described in literature (Table 1).

Table 1. Well-established and possible risk factors for carbapenemase-producing Klebsiella pneumoniae colonization and infection in clinical practice.

\begin{tabular}{lll}
\hline & Risk factors well established in literature & Possible risk factors \\
\hline Disease-related & Severe illness & High number of comorbidities \\
& High APACHE-II score & Chronic disease \\
& Need for mechanical ventilation & Immunodeficiency \\
& Respiratory failure & \\
\hline Healthcare-related & Prior hospitalization & Internal Medicine or Geriatric Unit admission \\
& Intensive Care Unit admission & Prior antibiotic exposure \\
& Prior therapy with carbapenems & Nursing home residency \\
& & Prolonged bed rest \\
& & High number of medical devices \\
& & High number of diagnostic invasive procedures \\
\hline Patient-related & & Advanced age \\
& & Frailty \\
& & Movement disability \\
\hline
\end{tabular}


Moreover, frail elderly usually have a different fecal microbiota composition than adults. ${ }^{23,24}$ This may cause a lower bacterial competition at an intestinal level, making rectal colonization easier for CPKP strains. In fact, once a CPKP-positive patient is discharged from hospital, a normal gut microbiota is restored as he or she recovers, and therefore CPKP can be spontaneously eradicated. Thus one may hypothesize that restoring a normal gut flora through probiotics might be an effective way to treat CPKP colonization and to prevent systemic infection onset.

\section{Our experience}

In our clinical experience at Parma University Hospital we observed 133 cases of CPKP positivity from August 2011 to May 2012. In most cases patients were frail elderly with many comorbidities admitted to internal medicine wards. Rectal swab performed for epidemiologic surveillance was the most frequent means of detection, so that simple enteric colonization was more common than actual infection. However, when infection occurred, it was extremely serious with development of a sometimes fatal septic shock.

In a subgroup of 36 CPKP-positive patients we also carried out a pilot study to detect the possible role of high-dose symbiotic probiotic in achieving CPKP eradication. Patients randomly received usual care alone (18 patients) or usual care plus a common commercial symbiotic supplement [5 high-dose $\left(24 \times 10^{9}\right.$ colony forming units) probiotic strains alive and psyllium fiber, 18 patients] for 14 days. Patients in both treatment and control group did not differ for general baseline features, even if patients who had developed a CPKP-related infection before randomization were $26 \%$ in treatment group and $6 \%$ in control group $(\mathrm{P}=0.186)$. In the treatment group we observed a significantly higher incidence of rectal swab negativization than in control group ( $53 \%$ vs $12 \%, \mathrm{P}=0.0094, \chi^{2}$ test). Mortality was not statistically different $(31 \%$ treatment group $v s 12 \%$ control group, $\mathrm{P}=0.236$ ). These preliminary results seem to confirm the assumption that gut microbiota is crucial in CPKP colonization in elderly patients and that probiotic therapy may be effective for eradication.

\section{Conclusions}

As our experience confirms, carbapenemase-producing $K$. pneumoniae is becoming a major health concern also in internal medicine and geriatric settings, especially for those patients who already have serious diseases and a complex clinical course. Raising clinicians' awareness about the importance of CPKP screening and management will be crucial to limit the pandemic spread, especially in endemic countries like Italy. We also hope that further research will clarify risk factors for CPKP colonization and the possible role of gut microbiota in the onset and management of the infection.

\section{References}

1. Peleg AY, Hooper DC. Current concepts: hospital-acquired infections due to Gram-negative bacteria. N Engl J Med 2010;362:1804-13.

2. Munoz-Price LS, Poirel L, Bonomo RA, et al. Clinical epidemiology of the global expansion of Klebsiella pneumoniae carbapenemases. Lancet Infect Dis 2013; 13:785-96.

3. Nordmann P, Cuzon G, Naas T. The real threat of Klebsiella pneumoniae carbapenemase-producing bacteria. Lancet Infect Dis 2009;9:228-36.

4. Gaibani P, Ambretti S, Farruggia P, et al. Outbreak of Citrobacter freundii carrying VIM-1 in an Italian hospital, identified during the carbapenemases screening actions, June 2012. Int J Infect Dis 2013;17:e714-7.

5. D'Arezzo S, Principe L, Capone A, et al. Changing carbapenemase gene pattern in an epidemic multidrug-resistant Acinetobacter baumannii lineage causing multiple outbreaks in central Italy. J Antimicrob Chemother 2011; 66:54-61.

6. Yirgit H, Queenan AM, Anderson GJ, et al. Novel carbapenem-hydrolyzing $\beta$-lactamase, KPC-1, from a carbapenem-resistant strain of Klebsiella pneumoniae. Antimicrob Agents Chemother 2001;45:1151-61.

7. Lomaestro BM, Tobin EH, Shang W, Gootz T. The spread of Klebsiella pneumoniae carbapenemase-producing K. pneumoniae to upstate New York. Clin Infect Dis 2006; $43: \mathrm{e} 26-8$.

8. Giani T, D'Andrea MM, Pecile P, et al. Emergence in Italy of Klebsiella pneumoniae sequence type 258 producing KPC-3 carbapenemase. J Clin Microbiol 2009; 47:3793-4.

9. Zarkotou O, Pornaras S, Tselioti P, et al. Predictors of mortality in patients with bloodstream infections caused by KPC-producing Klebsiella pneumoniae and impact of appropriate antimicrobial treatment. Clin Microbiol Infect 2011;17:1798-803.

10. Mouloudi E, Protonotariou E, Zagorianou A, et al. Bloodstream infections caused by metallo- $\beta$-lactamase/Klebsiella pneumoniae carbapenemase-producing $\mathrm{K}$. pneumoniae among intensive care units in Greece: risk factors for infection and impact of type of resistance on outcomes. Infect Contol Hosp Epidemiol 2010;31: 1250-6.

11. Daikos GL, Markogiannakis A. Carbapenemase-producing Klebsiella pneumoniae: (when) might we still consider treating with carbapenems? Clin Microbiol Infect 2011;17:1135-41.

12. Tumbarello M, Viale P, Viscoli C, et al. Predictors of mortality in bloodstream infections caused by Klebsiella pneumoniae carbapenemase-producing K. pneumoniae: importance of combination therapy. Clin Infect Dis 2012;55:943-50.

13. Qureshi ZA, Paterson DL, Potoski BA, et al. Treatment outcome of bacteremia due to KPC-producing Kleb- 
siella pneumoniae: superiority of combination antimicrobial regimens. Antimicrob Agents Chemother 2012; 56:2108-13.

14. Munoz-Price LS, Hayden MK, Lolans K, et al. Successful control of an outbreak of Klebsiella pneumoniae carbapenemase-producing K. pneumoniaeat a long-term acute care hospital. Infect Control Hosp Epidemiol 2010;31:341-7.

15. Nordmann P, Gniadkowski M, Giske CG, et al. Identification and screening of carbapenemase-producing Enterobacteriaceae. Clin Microbiol Infect 2012;18:432-8.

16. Gasink LB, Edelstein PH, Lautenbach E, et al. Risk factors and clinical impact of Klebsiella pneumoniae carbapenemase-producing K. pneumoniae. Infect Control Hosp Epidemiol 2009;30:1180-5.

17. Papadimitriou-Olivgeris M, Marangos M, Fligou F, et al. Risk factors for KPC-producing Klebsiella pneumoniae enteric colonization upon ICU admission. J Antimicrob Chemother 2012;67:2976-81.

18. Correa L, Dalla Valle Martino M, Siqueira I, et al. A hospital-based matched case-control study to identify clinical outcome and risk factors associated with carbapenem-resistant Klebsiella pneumoniae infection. BMC Infect Dis 2013;13:80.
19. Gaibani P, Ambretti S, Berlingeri A, et al. Rapid increase of carbapenemase-producing Klebsiella pneumoniae strains in a large Italian hospital: surveillance period 1 March-30 September 2010. Euro Surveill 2011;16: pii19800.

20. Giani T, Pini B, Arena F, et al. Epidemic diffusion of KPC carbapenemase-producing Klebsiella pneumoniae in Italy: results of the first countrywide survey, 15 May to 30 June 2011. Euro Surveill 2013;18:pii20489.

21. Gagliotti C, Ciccarese V, Sarti M, et al. Active surveillance for asymptomatic carriers of carbapenemase-producing Klebsiella pneumoniae in a hospital setting. J Hosp Infect 2013;83:330-2.

22. Franceschi C, Capri M, Monti D, et al. Inflammaging and anti-inflammaging: a systemic perspective on aging and longevity emerged from studies in humans. Mech Ageing Dev 2007;128:92-105.

23. Van Tongeren SP, Slaets JPJ, Harmsen HJM, Welling GW. Fecal microbiota composition and frailty. Appl Environ Microbiol 2005;71:6438-42.

24. Biagi E, Nylund L, Candela M, et al. Through ageing, and beyond: gut microbiota and inflammatory status in seniors and centenarians. PLoS One 2010;5:e10667. 